\title{
TOWARDS UNDERSTANDING SCENE TRANSITION TECHNIQUES IN IMMERSIVE 360 MOVIES AND CINEMATIC EXPERIENCES
}

\author{
An Undergraduate Research Scholars Thesis \\ by \\ SEYED KASRA RAHIMI MOGHADAM \\ Submitted to the Undergraduate Research Scholars program at \\ Texas A\&M University \\ in partial fulfillment of the requirements for the designation as an
}

UNDERGRADUATE RESEARCH SCHOLAR

Approved by Research Advisor:

Dr. Eric D. Ragan

May 2017

Major: Computer Science 


\section{TABLE OF CONTENTS}

Page

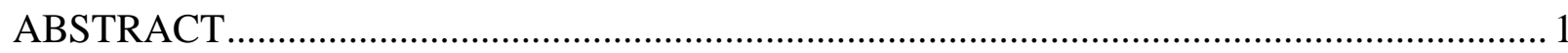

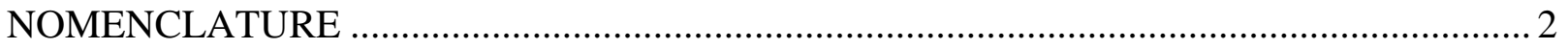

\section{CHAPTER}

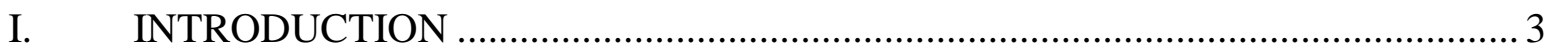

II. $\quad$ RELATED WORKS ............................................................................... 5

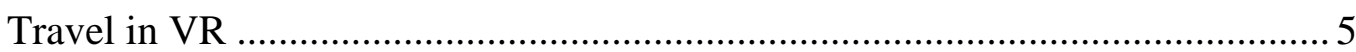

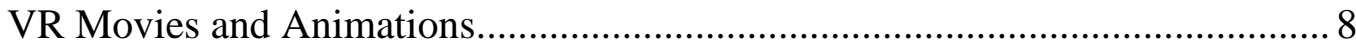

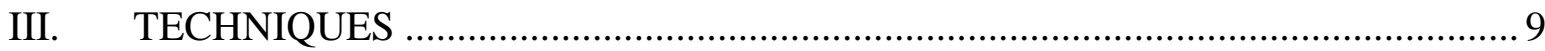

Teleportation (Instant Scene Change)................................................... 9

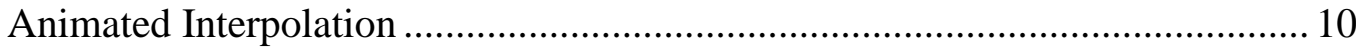

Pulsed Interpolation .............................................................................. 10

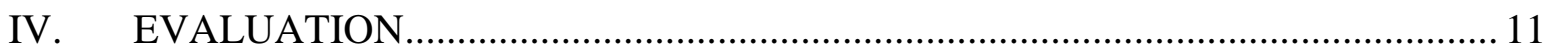

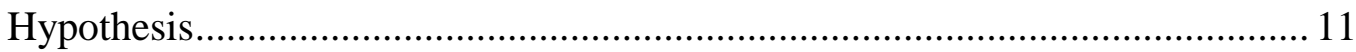

Experimental Environment and Task....................................................... 12

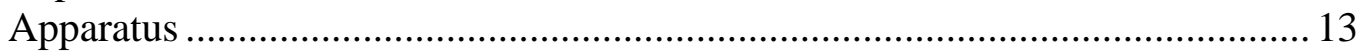

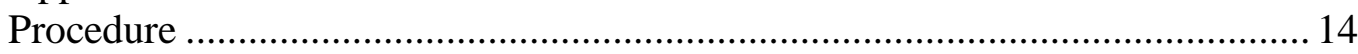

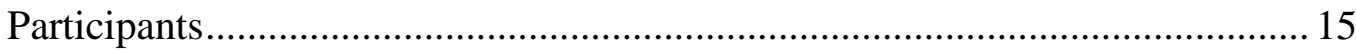

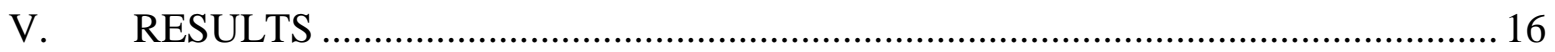

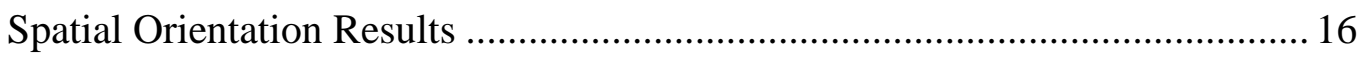

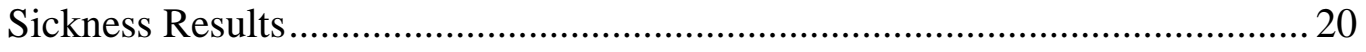

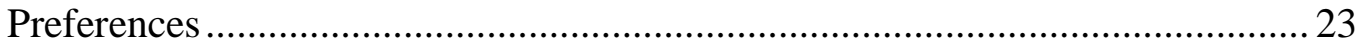

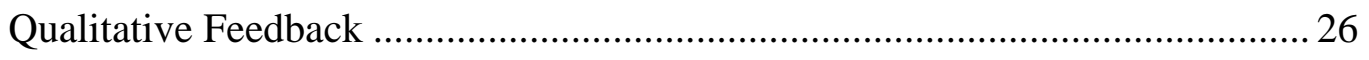

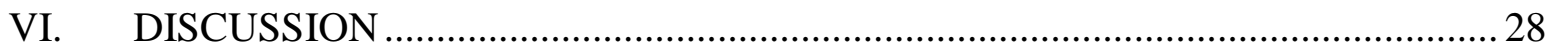

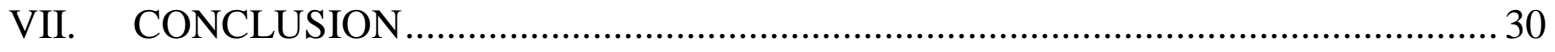

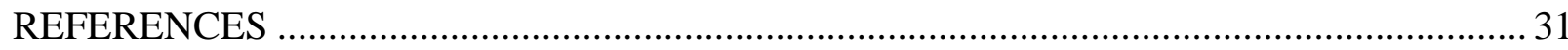




\begin{abstract} Experiences

\author{
Seyed Kasra Rahimi Moghadam \\ Department of Computer Science and Engineering \\ Texas A\&M University
}
Research Advisor: Dr. Eric D. Ragan
Department of Visualization
Texas A\&M University

Towards Understanding Scene Transition Techniques in Immersive 360 Movies and Cinematic

Many researchers have studied methods of effective travel in virtual environments, but little work has considered scene transitions, which may be important for virtual reality experiences like immersive 360-degree movies. In this research, we designed and evaluated three different scene transition techniques (Teleportation, Animated Interpolation, and Pulsed Interpolation) in two environments. Our goal was to compare these techniques and their variations to find out which works the best in different situations. We designed an experiment to evaluate how different techniques influenced sickness and the ability to maintain spatial awareness of object locations in dynamic virtual environments. With this approach, we first assessed the impact of different speeds, visual adjustments, and movement types for each of the three transition types. Next, we conducted a study comparing the best variations of each of the three techniques. Our results showed that the Teleportation technique caused the least sickness and the Animated technique was best for maintaining spatial awareness. 


\section{NOMENCLATURE}

$\begin{array}{ll}\text { HMD } & \text { Head Mounted Display } \\ \text { IQR } & \text { Interquartile Range } \\ \text { IVE } & \text { Immersive Virtual Environment } \\ \text { VE } & \text { Virtual Environment } \\ \text { VR } & \text { Virtual Reality }\end{array}$




\section{CHAPTER I}

\section{INTRODUCTION}

With the recent release of the commercial head-mounted displays (HMDs) such as Oculus Rift, HTC Vive, Samsung Gear VR, Google Cardboard, and PlayStation VR, interest in virtual reality for home entertainment is more than ever. Users can experience the spatial impression of an immersive virtual environment (IVE) using these technologies [14]. Much research has been done to enhance spatial orientation, minimize motion sickness, and increase the feeling of presence in the virtual environment (VE) for the people using them.

Travel and view control is a core topic that has been researched extensively in VE research $[1,2,3,4,5,6,7,8,12,13,15]$. Bowman et al. [4] define travel as the control of user viewpoint motion in. They state in other work [5] that travel is the most common interaction in IVE applications after simple head rotation, but it is generally not a primary goal itself; rather, it is used to help the user complete other more important tasks. Therefore, it is important to facilitate cognitively simple and easy-to-use techniques for traveling in IVEs.

While much work has been done in regards to travel in IVEs, there are not many studies done on scene transitions in IVEs. The main difference between traveling and scene transition in IVEs is that the viewpoint motion in traveling techniques is predominantly under the control of the user, whereas in scene transition techniques, the viewpoint motion is initiated and controlled by the system and the user has no control over it. Scene transition can be useful for long movements or view adjustments in many types of 3D applications. For example, transitions are found in video games to change levels, in movies for cuts and transitions between scenes and locations, and in other applications to show new perspectives within a $3 \mathrm{D}$ environment. Our 
work is also motivated by the common use of scene transitions in film and narrative experiences. Many types of traditional narrative experiences commonly rely on scene transitions to change locations or direct viewer attention. However, there is a lack of knowledge about the effects of different methods of scene transition on spatial orientation in the IVE and how much sickness different methods can cause. If not understood, a poor choice of transition could cause discomfort or disorientation to a viewer trying to follow a narrative experience in a 3D world. In this research, we conducted four controlled experiments to compare three different methods of scene transition in two different environments. Three of these experiments compared different variations of each technique and one of them compared all the techniques together. Through user testing, we collected metrics related to sickness, spatial orientation, and preference for participants as they kept track of moving objects in a dynamic virtual scene. 


\section{CHAPTER II}

\section{RELATED WORK}

In this section, we discuss prior research about travel techniques and immersive VR movies and experiences.

\section{Travel in VR}

In regards to spatial orientation and feeling of presence, research has shown benefits to realistic methods of travel, with real walking and turning outperforming less natural methods $[2$, $6,7,12,13,15]$. For example, Chance et al. [7] compared techniques that differed in their level of similarity to real walking in the physical world. They found that a technique more similar to real walking produced a better spatial orientation in subjects comparing to techniques in which self-motion was virtual and the subjects remained still while their view was being translated.

However, small workspaces and limited range of tracking sensors are the drawbacks of this method [2]. Therefore, methods for navigation in IVEs are needed to travel large distances without walking in the real world. Bolte et al. [2] developed a metaphor called "The Jumper Metaphor" that let the users travel short distances by walking, whereas if the user wanted to travel large distances, it predicted the location that the user wanted to end up and made her virtually jump to that position. Then, they compared the condition where this technique is present with two other conditions: the condition where the user is only allowed to walk and the jumper metaphor is not present and the condition where the jumper metaphor is replaced with teleportation. They observed that users were worse at map sketching after using teleportation compared to real walking and jumper metaphor which showed that they experienced disorientation using the teleportation technique. Moreover, the users preferred the smooth 
viewpoint animation of jumper metaphor to instant viewpoint change of teleportation, and they greater difficulty with the traveling task when using the teleportation technique.

Relevant to scene transitions, Freitag et al. [8] a method similar to teleportation that used portals move users to a different location and reorient them when they approached workspace boundaries. This technique enabled users to physically walk around to travel in the virtual world without colliding with the walls in the real world. They compared their portal technique with two other techniques in their experiment: point-and-fly (which enabled the user to fly in the scene when traveling) and teleportation. Point-and-fly technique proved to cause the most sickness in the subjects and there was no significant difference between the portal and teleportation techniques. They attributed this to the fact that these two techniques did not rely on a virtual motion that can cause simulator sickness due to conflicts of different senses. In contrast, loss of orientation was seen least often when the point-to-fly technique was used and most often when the portal technique was used which was because of the reorientations that were done on the subjects every time they went through a portal.

Regarding instant viewpoint change, Bowman et al. [5], during their research about comparing two metaphors (gaze-directed and hand-directed) for travel, concluded that abrupt change of view is disorienting and smooth transitions showed better results for spatial awareness. Although this jumping technique they used in their experiment was better in regards to accuracy and speed, it proved to cause disorientation for the subjects. In another research, Bowman et al. [3] studied the effects of velocity and acceleration on spatial awareness. They found out that there was no significant difference in spatial awareness with different levels of velocity or acceleration, except when the velocity was infinite (the user was instantly teleported). This teleportation caused great disorientation in subjects. Tomlinson et al. [14] used two different 
scene transition methods while researching their virtual cinematographer. The Cut method causes the camera to immediately go to the new position and Whip-pan, swoops the camera through the space rapidly when changing its position. However, the study did not make any comparisons between these two methods in respect to the sickness they might cause and their effects on spatial orientation.

In another study, Bowman et al. [4] studied the differences of system-automated travel and user-controlled travel with respect to spatial awareness by asking the subjects to complete a task where they had to travel through a corridor followed by a pointing task. Three objects were placed in that corridor and in the end, the subjects were asked to point toward one of those objects. Interestingly, they did not find any significant differences between the performances of the travel techniques. The system-automated technique produced a slightly lower average error, but the differences were not significant. They compared these two techniques to the real world situation of traveling by car. The driver's condition is the same as the user-controlled traveling technique, while the passenger's condition is similar to the system-automated technique. The driver can have a better spatial orientation since she must attend to the spatial information in steering the car. On the other hand, if the passenger is observant enough and carefully acquires spatial knowledge while sitting in the car, she might be able to maintain a better spatial orientation since she is not distracted with the task of driving the car.

Furthermore, Vasylevska et al. [16] introduced a new elevator metaphor for vertical navigation in IVEs. They compared their technique to existing flying and teleportation techniques with respect to spatial presence, comfort, and real world awareness. Their results showed that teleport had the lowest score for spatial presence while flying and elevator techniques did not have a significant difference. For the comfort and real world awareness, 
flying had the lowest scores, but there were no significant differences between teleport and elevator techniques.

\section{VR Movies and Experiences}

After the commercial release of VR headsets, a number of VR movies and animated experiences have been produced. Most of them are stationary experiences with no scene transitions. For example, Oculus Story Studios [11] uses no scene transitions in their animations Henry and Lost. However, there are some movies that use system-automated viewpoint motions for scene transition. We will introduce some of them and will examine their scene transition techniques.

Mr. Robot: Virtual Reality Experience uses both instant scene change and interpolated transition techniques. It uses instant scene change for cuts between different locations, and it uses it both in a way that there is a black transition scene in between the two scenes and in a way where there is no black transition scene. It also uses interpolated transition to move the viewer's viewpoint around the scenes. Camp Life: Woodward also uses both these techniques in the same way. It also uses interpolated transition to simulate the feeling of riding a bike for the viewer.

Google Spotlight Stories' [9] Help uses interpolated transition to move the viewer around different locations. The camera follows the characters of this movie in their escape from a monster. The promotional movie, Rio 2016, on the other hand, only uses instant change technique to move the viewer between different locations and scenes. 


\section{CHAPTER III}

\section{TECHNIQUES}

In our research, we designed, implemented, and evaluated different scene transition techniques for viewpoint motion in short distances. Here, we describe the three techniques we studied.

\section{Teleportation (Instant Scene Change)}

The first technique is Teleportation, which involves an instant change in viewpoint position or rotation. The viewer will not see the process of viewpoint motion in this technique and she is instantly translated or rotated. We studied two different variations of this technique: Instant Change where the viewpoint changes state without any delays (Figure 1), and Fade to Black where there is a fade in/fade out to a black transition scene and then the viewer finds herself in the new state (Figure 2).
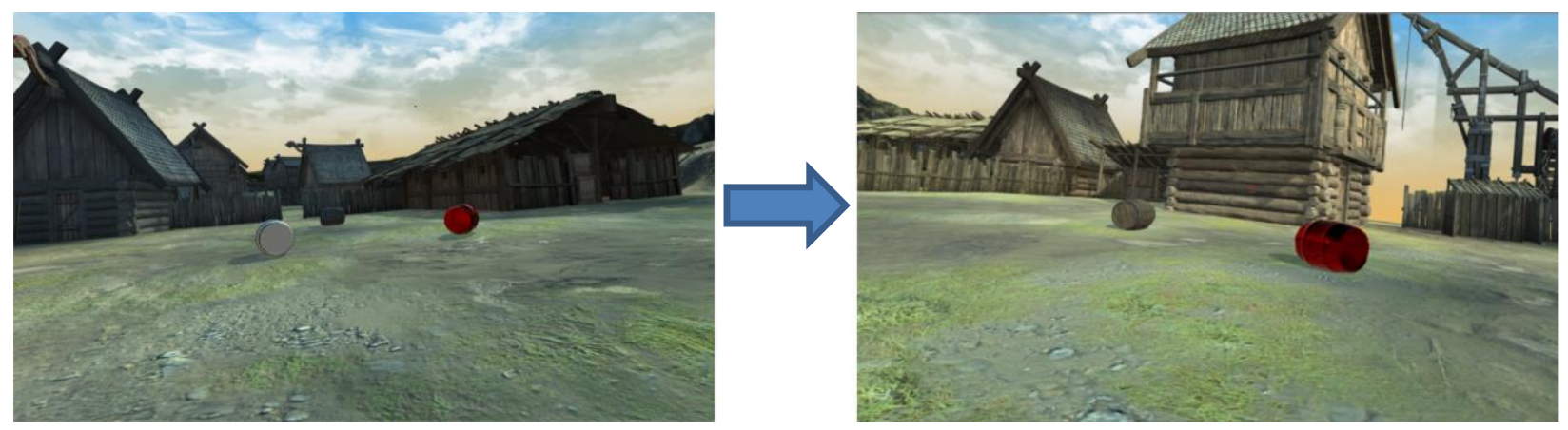

Figure 1: Teleportation technique (Instant Version)
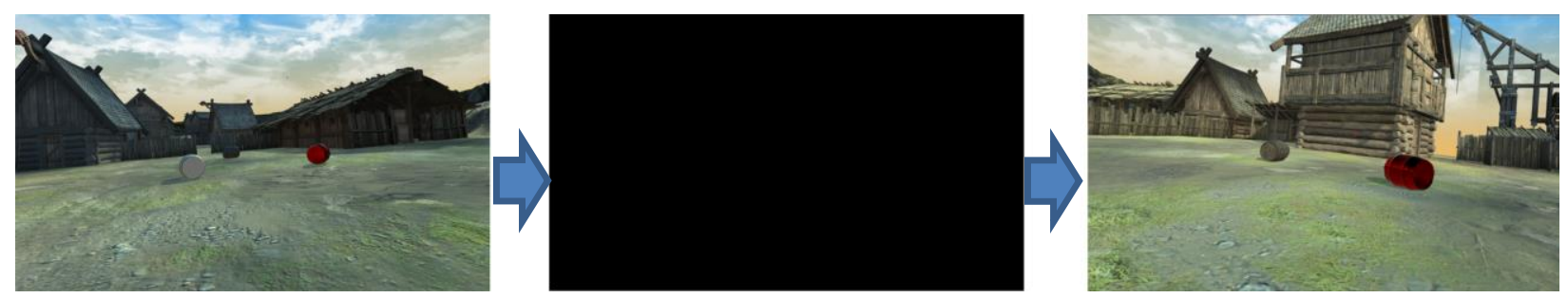

Figure 2: Teleportation technique (Fade to Black version) 


\section{Animated Interpolation}

The second technique is Animated Interpolation, which is a smooth viewpoint motion from one state to another. The viewer can observe the process of being translated or rotated to the new state. We studied three variations of this technique with different speeds for the transition: slow $(10 \mathrm{~m} / \mathrm{s})$, medium $(25 \mathrm{~m} / \mathrm{s})$, and fast $(50 \mathrm{~m} / \mathrm{s})$.

\section{Pulsed Interpolation}

The third technique is Pulsed Interpolation. Unlike the constant interpolation used in the previous technique, the pulsed technique fades the view in and out several times along the transition from one state to another (Figure 3). We also studied different variations of this technique with varying amounts of intermediate points between the start and end points. The number of intermediate points we tested for were 2 (lowest), 3 (middle), and 4 (highest).

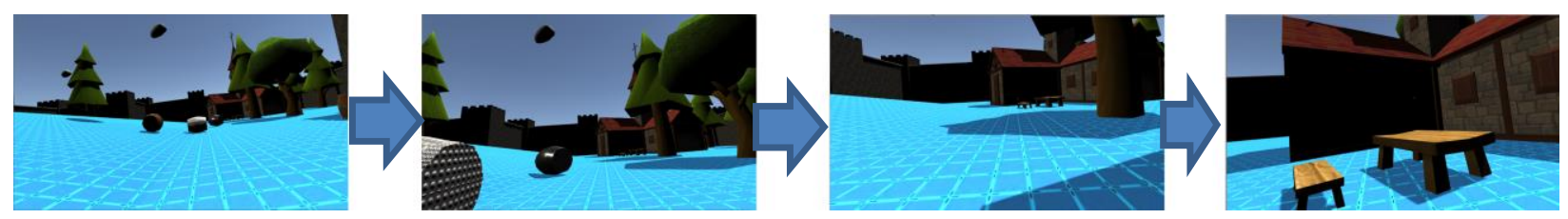

Figure 3: Pulsed Interpolation technique

In our evaluation of these different transition techniques, we also studied whether different techniques were better suited for different types of motions. To this end, our experiments also tested three different types of viewpoint changes: (1) only translational motion, (2) only rotational motion, and (3) both translation and rotation at the same time. 


\section{CHAPTER IV}

\section{EVALUATION}

We performed four controlled experiments to compare different scene transition techniques in terms of their effects on spatial orientation, sickness, and preference. In the first experiment, we tested different fade effects for the Teleportation technique. In the second experiment, we tested different speeds for the Animated interpolation technique. In the third experiment, we tested different numbers of intermediate points for the Pulsed interpolation technique. Lastly, we compared the Teleportation, Animated, and Pulsed techniques to each other.

\section{Hypothesis}

We expected that the Teleportation technique would cause the least sickness for the participants and the Animated interpolation techniques would be significantly better than Teleportation in regards to performance in the spatial awareness task. The Animated technique was the one that we thought would cause the most sickness because participants could see the whole process of moving from one position to another without having any control over the camera's speed and orientation. We expected the Pulsed interpolation technique to be somewhere in the middle, meaning in doing the spatial awareness task of the study, it would help participants perform better than Teleportation but worse than Animated, and it would cause less sickness than Animated but more sickness than Teleportation.

We also expected that participants with more 3D gaming experience would have better spatial awareness performance and would experience fewer sickness symptoms. Finally, we 
expected that gamer participants and non-gamer participants would prefer different techniques for scene transitions in VR.

\section{Experimental Environment and Task}

In our user study, participants tested different variations of the techniques in two simple scenes: (1) a Viking village with monotonous colors and (2) a cartoonish fort with high color contrast (see Figure 4).
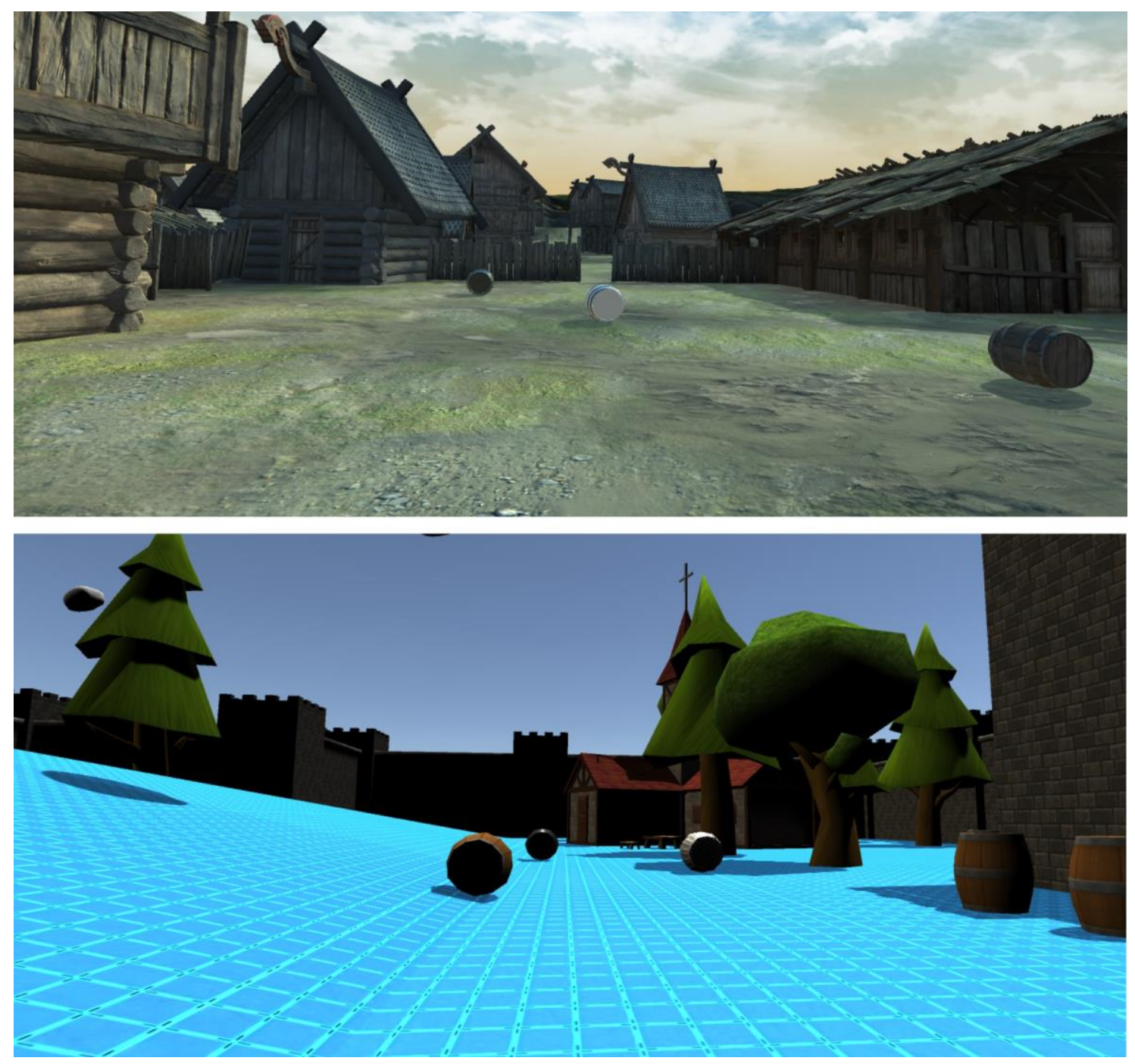

Figure 4: The two virtual environments used in the study.

We conducted a between-subjects study by varying three techniques between participants: teleportation, animated interpolation, and pulsed interpolation. Participants also 
experienced different variations of each technique. Variations included different types of viewpoint motions, speeds, and fade effects (as discussed in the Techniques section). The different variations were tested within subjects and each of the techniques was tested by 10 participants. After analyzing the data we collected from these studies, we chose the best variation of each technique (based on participants' performances and feedback) and conducted a withinsubject study where each participant tested all the three techniques in one session. The total number of people who participated in the combination study was 16.

To test the techniques and allow assessment of viewer ability to maintain spatial awareness during scene transitions, participants had to complete a simple object-tracking task involving three moving barrels. Participants watched the barrels moving in random directions and were told to focus on their positions. Then, a scene transition was initiated and the viewpoint state was changed to a new randomly selected state. After the transition, one of the barrels was missing, and participants were then asked to indicate the last position of that barrel using a red cursor that appeared in front of their viewpoint. The study had multiple sessions and the participants tested a different variation of the specific technique (as discussed in the techniques section) in each session (in the combination test, we switched between techniques instead of variations).

During a session, the task was repeated 3 times for each type of viewpoint change (as discussed in the techniques section) for a total of 9 times. Every time the participants finished one task, the program reappeared the disappeared barrel and changed the colors of barrels randomly for a new task. All the sessions were done in both the Viking village and the fort scenes. 


\section{Apparatus}

The study was run on 64-bit Windows 7 Professional. The computer had a 3.6 Ghz Quad Core processor and a GeForce GTX 980 4GB graphics processing unit. Participants viewed the environments in an Oculus Rift (consumer version 1.0). Positional and rotational head-tracked viewing was enabled through the Oculus Rift's Constellation tracking system. Participants sat in a rotating chair for the study and they could turn freely. The study application was implemented in Unity3D version 5.4.1f1.

\section{Procedure}

The study was reviewed and approved by our university's Institutional Review Board (IRB). Participants first received an introduction to the study goals and procedures, and they were asked to read and sign a consent form with detailed information about the study and the possible risks involved. Then, using a background questionnaire, we gathered data about each participant's age, gender, occupation, and prior experiences with video games and virtual reality. We then described the task they had to complete during the experiment and ran a practice run for them to get familiar with what they had to do. Whenever they felt they were ready for the experiment, we stopped the practice run and ran the main experiment.

Participants could take a 2-minute break in between each session. In addition, they could take a longer 5-minute break when they were done with all the sessions for the first scene and before starting the experiment for the second scene. At the very beginning of the study and after

each session of study, the participants were asked to complete a simulator sickness questionnaire based on Kennedy's SSQ [10] and rank sickness symptoms on a scale from 0 to 3 (none, slight, moderate, and severe). 
As another dependent measure, we recorded the angular offset between the correct direction of the removed barrel and the direction where participants were looking. This metric was used to assess spatial understanding of the dynamic scene after the transition.

Lastly, participants completed a post-study questionnaire about sickness, preferred technique variations, and ease of task completion. Also, we asked for general feedback about the techniques in an informal interview at the very end of the study session. The entire procedure took approximately 45-60 minutes.

\section{Participants}

46 participants completed the study: 10 for each technique ( 7 male and 3 female for each) for a total of 30 and 16 for the combination study where we tested all the three techniques in a within-subject study (15 male and 1 female). All were university students and ages ranged from 19 to 30. All participants had a good knowledge of computers and technology, and their selfreported weekly computer usage ranged between 40 to 80 hours. We had a mixture of gamers and non-gamers in our participants. About $60 \%$ of our participants identified themselves as avid gamers. Moreover, the participants reported mixed levels of experience with VR, and almost half the participants had tried a VR experience at least once before. 


\section{CHAPTER V}

\section{RESULTS}

In this section, we report the quantitative and qualitative data we collected in the studies we conducted for each technique and their variations, as well as in the combination study where we tested all the three techniques in a within-subject study.

Quantitative results are shown graphically using standard box-and-whisker plots. The box represents the interquartile range (IQR) with a horizontal band for the median. Each whisker extends to the most extreme value falling within an additional half-IQR beyond the IQR (in both directions), and outlier dots show values outside this range.

For the results from the simulator sickness questionnaire, we used Kennedy et al.'s [10] formula to calculate the total severity of nausea, oculomotor, and dizziness symptoms. Participants could choose between "None", "Slight", "Moderate", and "Severe" options for each symptom in the questionnaire and those options corresponded to numbers $0,1,2$, and 3 respectively. We used those numbers in the mentioned formula to get the total severity.

In the post-study experience questionnaire, participants could rank different characteristics of the scene transition techniques from 1 to 10 . We informed them that "l" always corresponds to least desirable or least significant and " 10 " always corresponds to most desirable or most significant.

\section{Spatial Orientation Results}

We analyzed the results of the pointing assessment as the objective measure of spatial orientation from the experiment. The outcomes from trials for each movement type of each variation block were averaged together for the studies that tested different variations of one 
technique, and the outcomes from trials for each movement type of each technique block were averaged together for the combination study where we tested all the three techniques.

For the Teleportation technique, we found that participants did better with the instant variation when the movement type was "translation only", while they did slightly better with the fade-to-black variation for both the "rotation only" and "both translation and rotation" movement types (see Figure 5).

\section{Overall Average Pointing Error for Teleport}

180

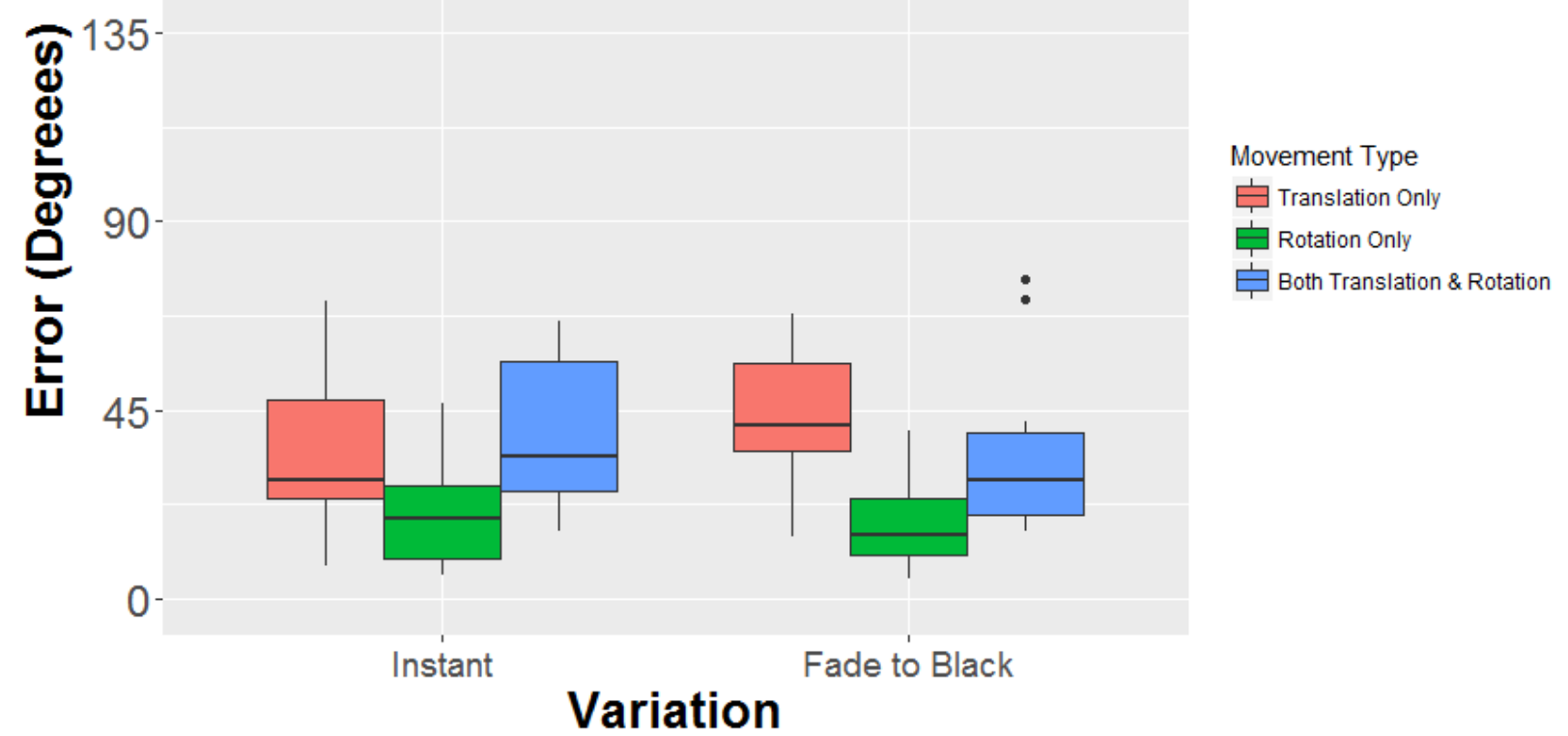

Figure 5: Average pointing error for different variations of the Teleportation technique.

For the Animated technique, it was easier for the participants to locate the disappeared barrel with the slow variation for the "translation only" and "both translation and rotation" movement types. For the "rotation only" movement type, they did better with the fast variation, which was expected because when rotating faster, the barrels were out of participants' field of 
view for a shorter amount of time, so they could recall the position of disappeared barrel easier than the other two variations (see Figure 6).

\section{Overall Average Pointing Error for Animated} 180

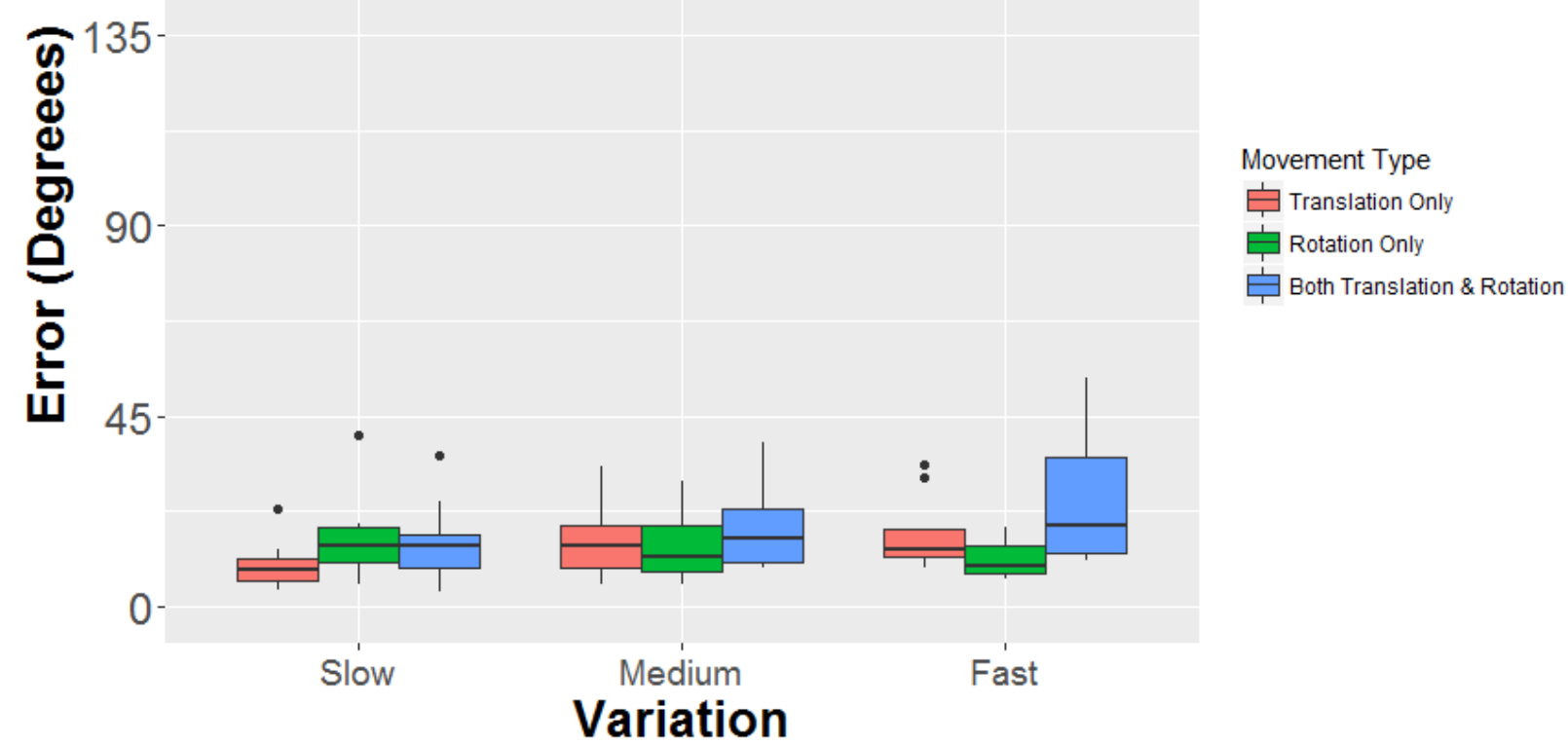

Figure 6: Average pointing error for different variations of the Animated technique.

The results for the Pulsed technique were very close and no significant differences were detected between participants' performance using different variations of the technique. It was only the variation with the medium number of intermediate points that showed better results for the "both translation and rotation" movement type than the other two variations (see Figure 7). Results were similar for the other two movement types. 


\section{Overall Average Pointing Error for Pulsed}

180

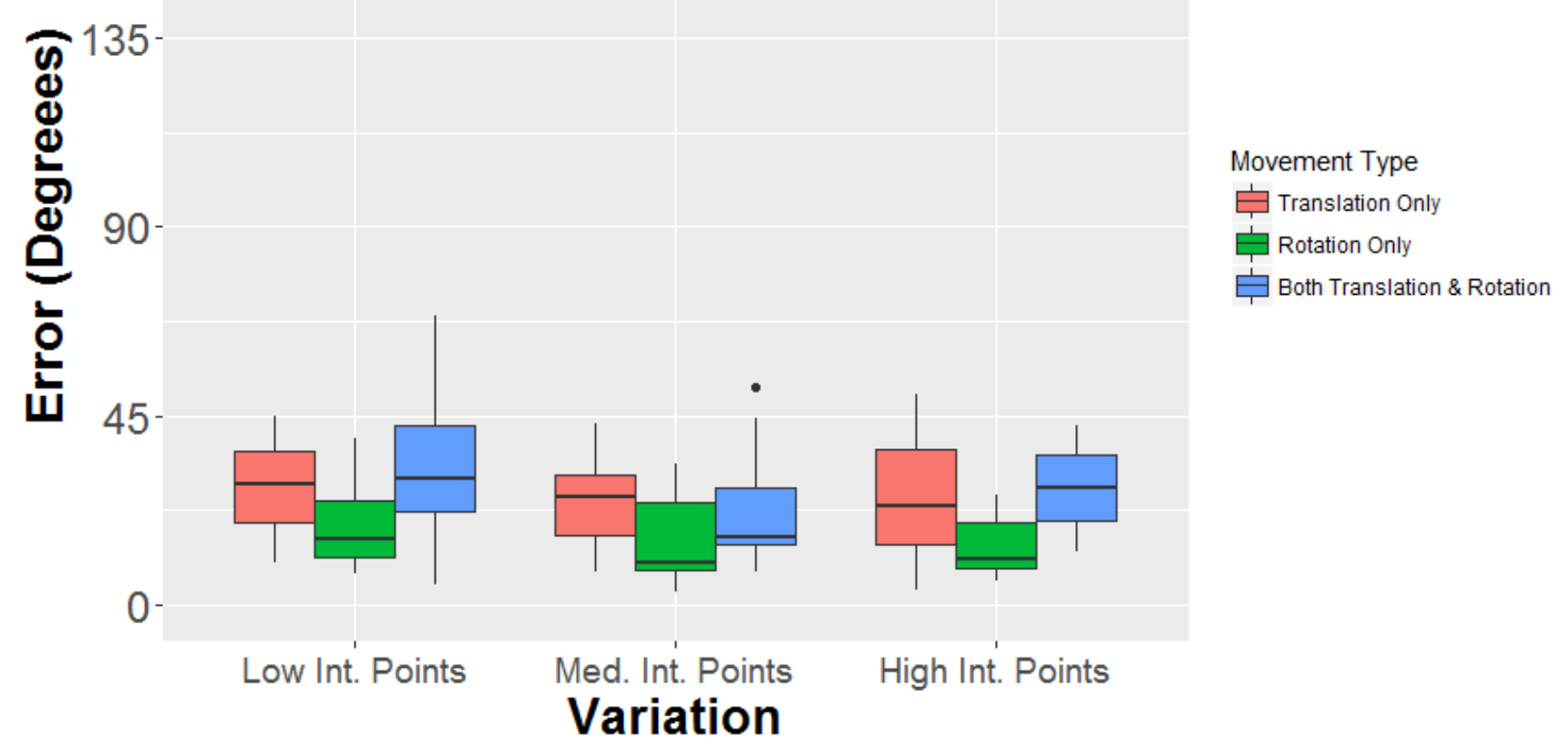

Figure 7: Average pointing error for different variations of the Pulsed technique.

The differences were greater for the combination study where we tested all the three techniques in a within-subject study. For the "translation only" and "both translation and rotation" movement types, the Animated technique showed the best results and Pulsed showed better results than Teleportation. These results aligned with our hypothesis. However, for the "rotation only" movement type, the results were almost the same (see Figure 8). Note that for the combination study we chose the instant variation for Teleportation, the slow speed for Animated, and the variation with the medium number of intermediate points for Pulsed based on participants' performance in the previous three studies where we tested each technique and its variations on its own. 


\section{Overall Average Pointing Error for Combined}

\section{0}

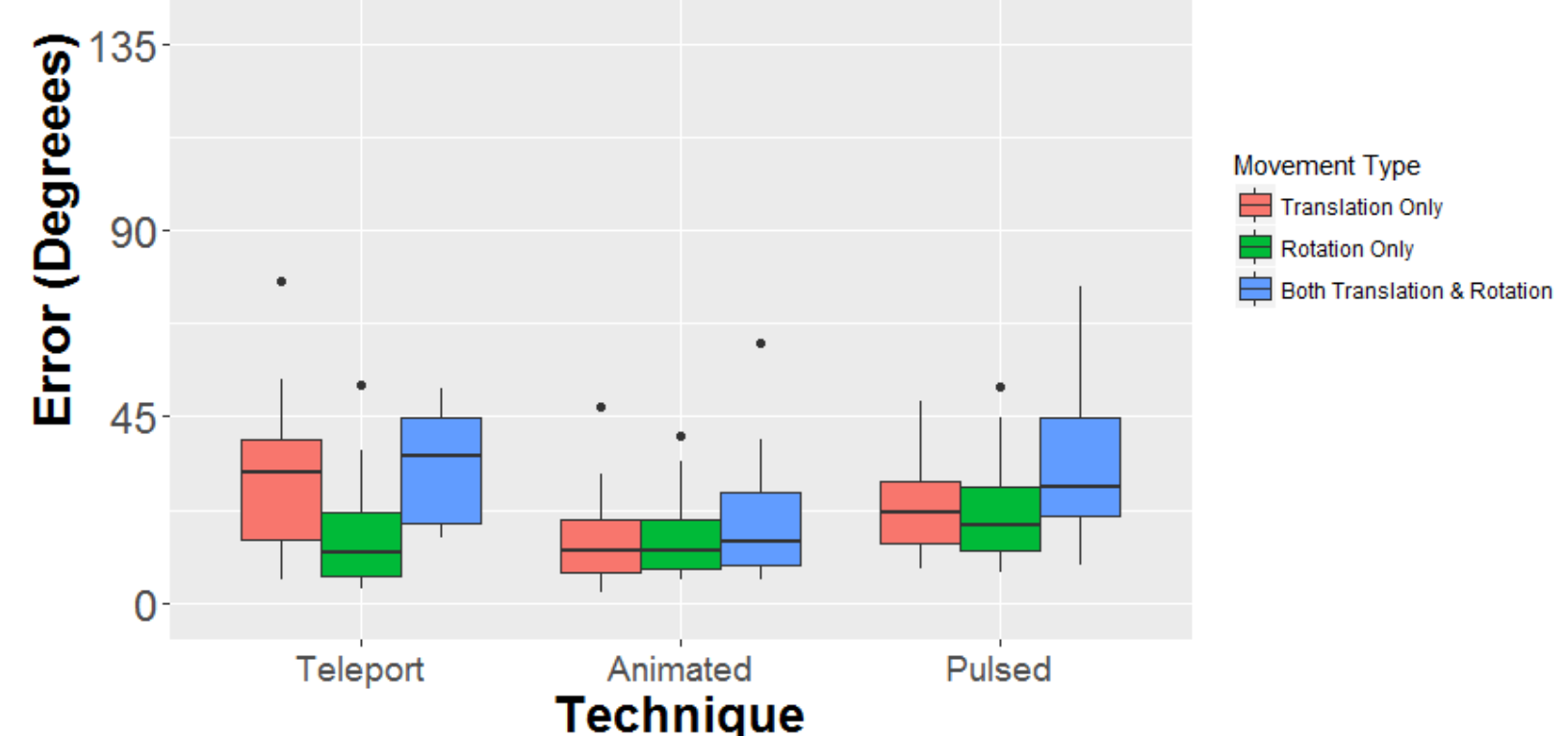

Figure 8: Average pointing error for different techniques in the combination study.

\section{Sickness Results}

As mentioned in the evaluation section, participants completed a simulator sickness questionnaire before starting the study and after each session of the study. After calculating the total severity of sickness symptoms, we observed that average sickness levels were relatively low over the entire study (see Figure 9). 

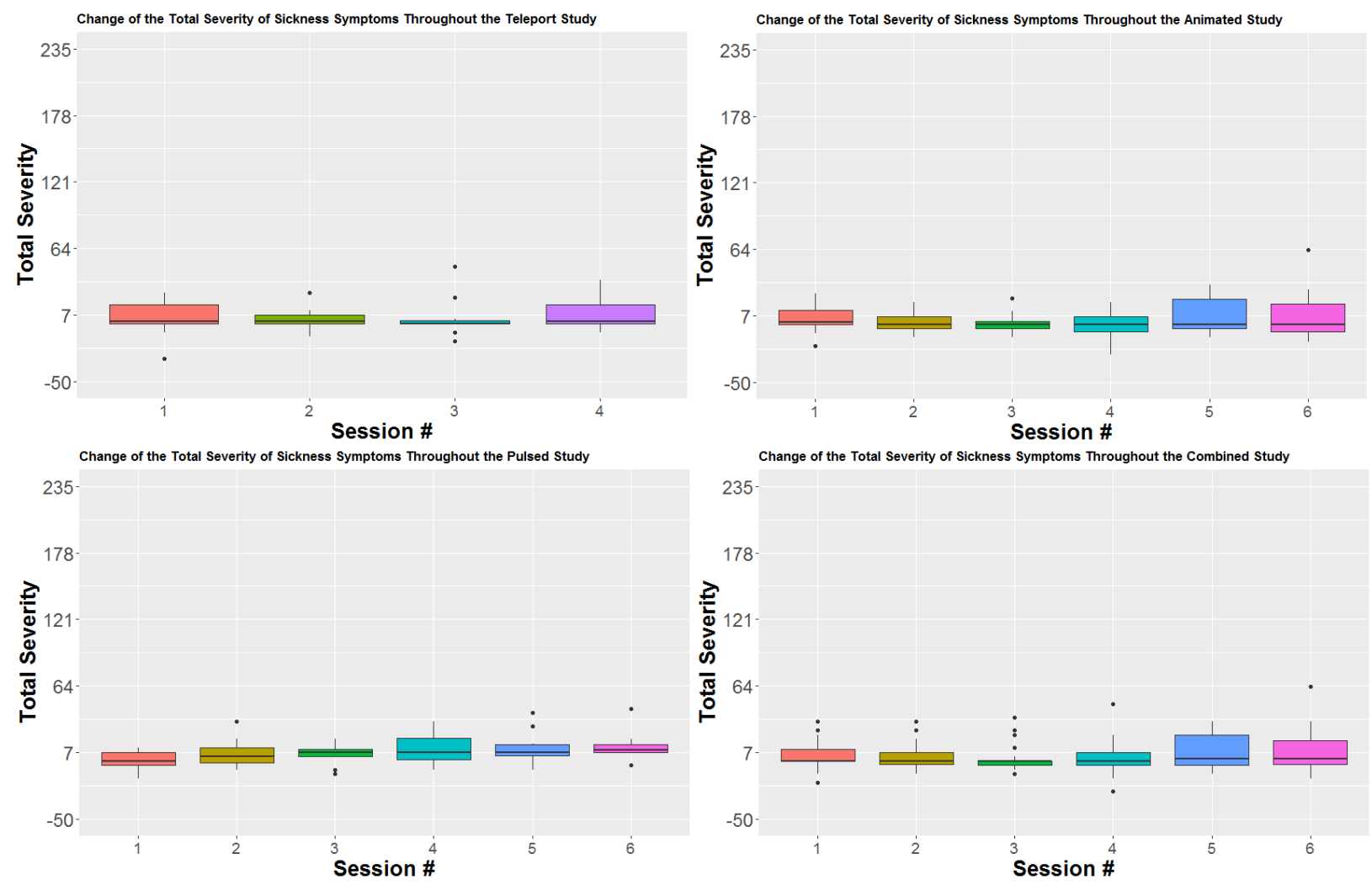

Figure 9: Change of the total severity of sickness symptoms over the entire study for all the 4 experiments we conducted. The "Session \#" on the $x$-axis corresponds to the session they completed the SSQ after. The numbers given to sickness symptoms in the initial SSQ by the participant are subtracted from all the corresponding numbers in the SSQs that were completed during the study.

Moreover, we asked the participant to rate the dizziness and the sickness they felt using each technique or the variation of each technique in the post-study experience questionnaire. They rated their dizziness and sickness on a scale from 1 to 10.

For the Teleportation technique, both sickness and dizziness rankings were very low as expected (see Figure 10). As it can be seen in the box plot, the median score for sickness for both the instant and fade-to-black variations was a magnitude of 1, which basically means no sickness since that is the lowest possible score participants could give to sickness caused by the technique. For dizziness, the median score was 2 for both variations and the fade-to-black variation had worse ratings than the instant variation in general. 

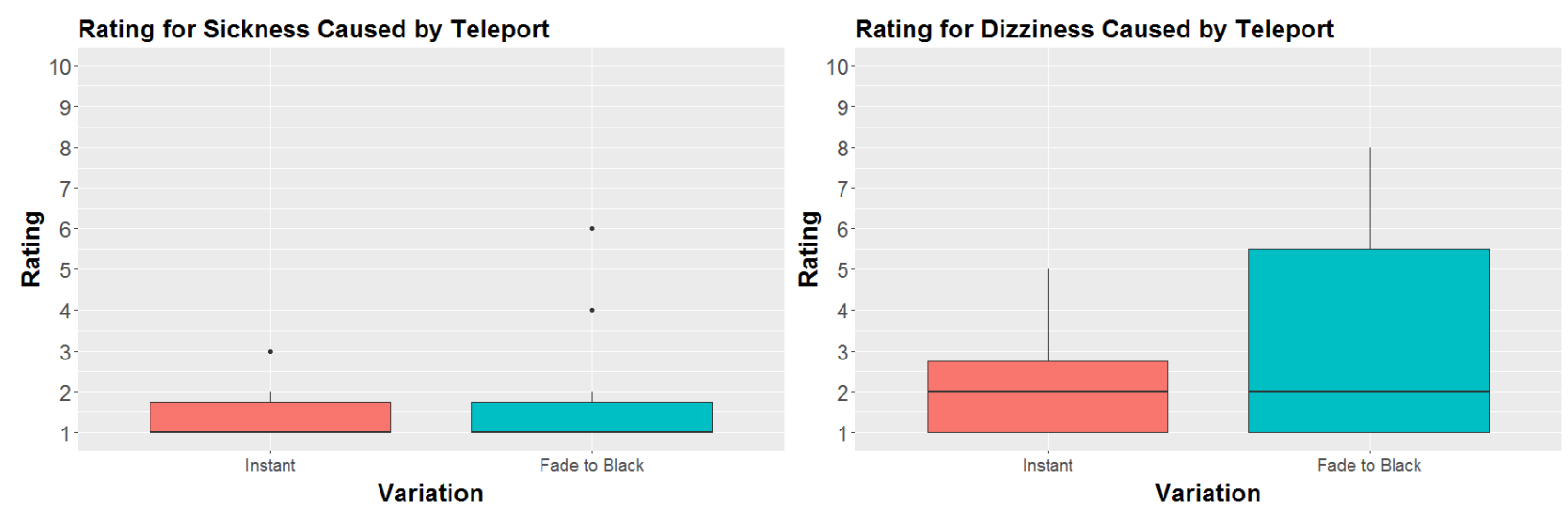

Figure 10: The ratings for the sickness and dizziness caused by different variations of the Teleportation technique.

For the Animated technique, the median score for sickness was around 2 and the median score for dizziness was 4 for all the three variations of the technique. The box plot for dizziness showed that the fast variation caused the most sickness for participants (see Figure 11).
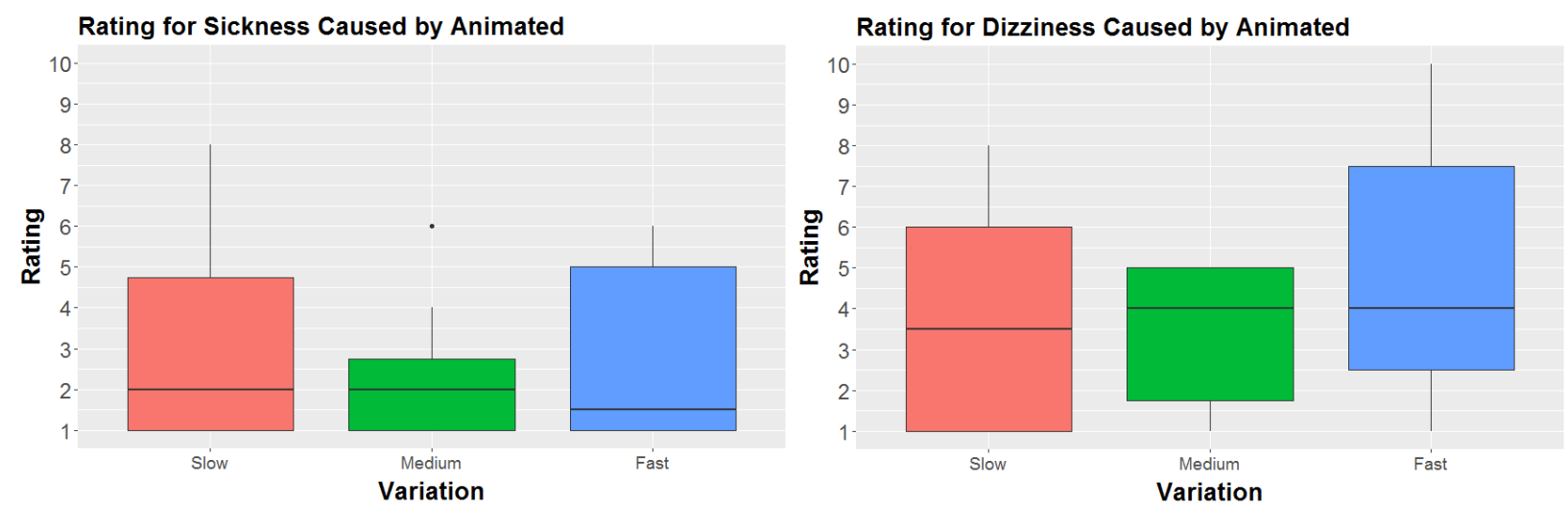

Figure 11: The ratings for the sickness and dizziness caused by different variations of the Animated technique.

The median score for the sickness caused by the variation with a low number of intermediate points of the Pulsed technique was 1 and it showed that it was close to the Teleport technique and caused a very low level of sickness or nothing at all. The rating was not as good for the dizziness, though. The median score for the dizziness caused by it was close to 3 . For the variation with medium number of intermediate points, the median score for sickness was 2 and it was worse than the first variation, but the median score for dizziness was 2.5 which was better. The third variation which had the most number of intermediate points was much worse than the 
first two with a median score of 3.5 for sickness and a median score of 4.5 for dizziness (see Figure 12). These results showed that the Pulsed technique caused less sickness with fewer numbers of intermediate points and it might not be a good idea to use it with high numbers of intermediate points.
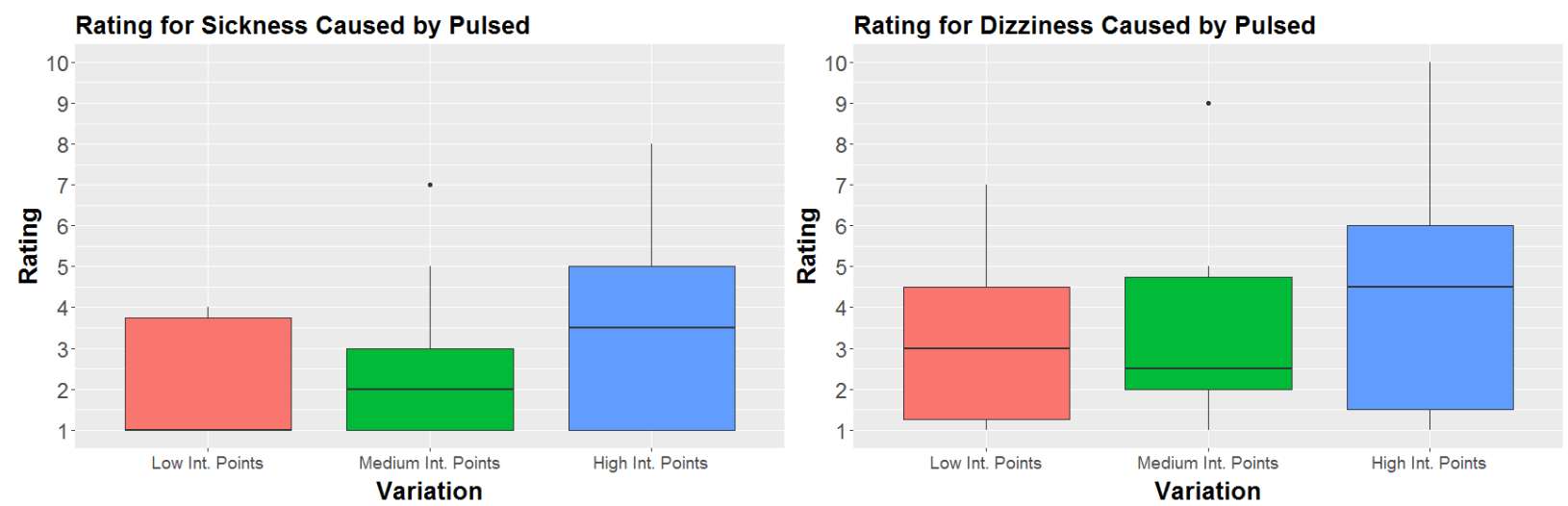

Figure 12: The ratings for the sickness and dizziness caused by different variations of the Pulsed technique.

The results of the combination study clearly show that the Animated technique caused the most sickness and dizziness (see Figure 13). The participants who tested all the three techniques found the Teleport technique to cause the least sickness and dizziness and they rated the Pulsed technique as the second best.
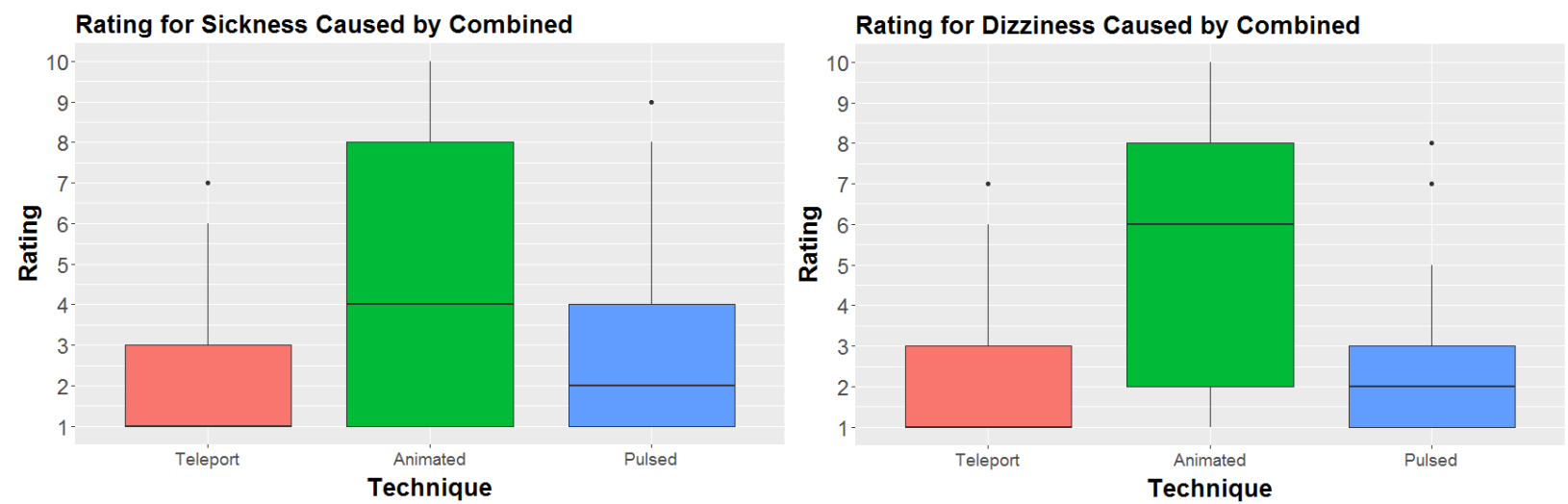

Figure 13: The ratings for the sickness and dizziness caused by different techniques in the combination study. 


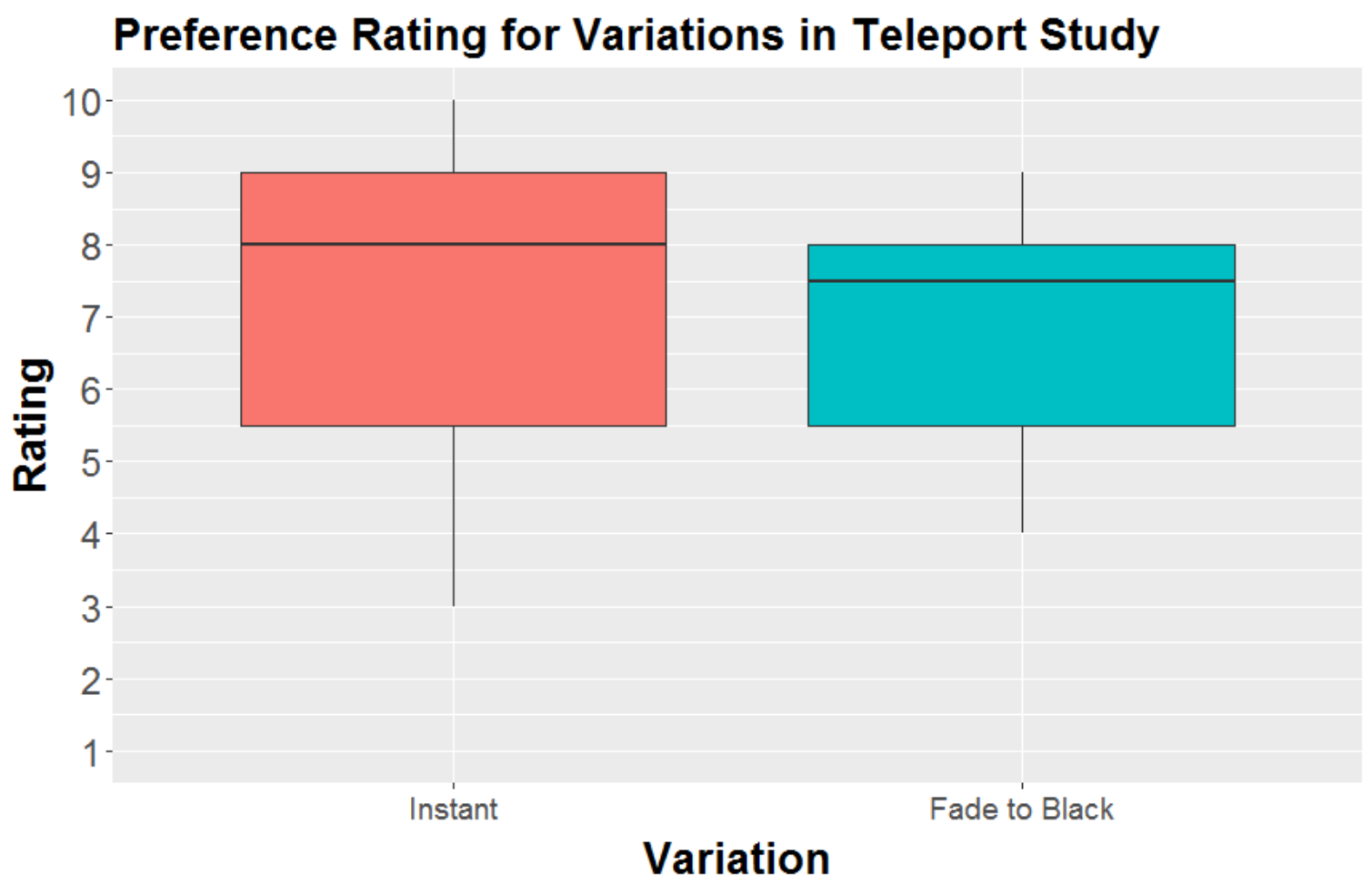

Figure 14: Preference ratings for different variations of the Teleport technique. The ratings were very close and the instant variation was rated slightly higher than the fade-to-black variation.

\section{Preference Rating for Variations in Animated Study}

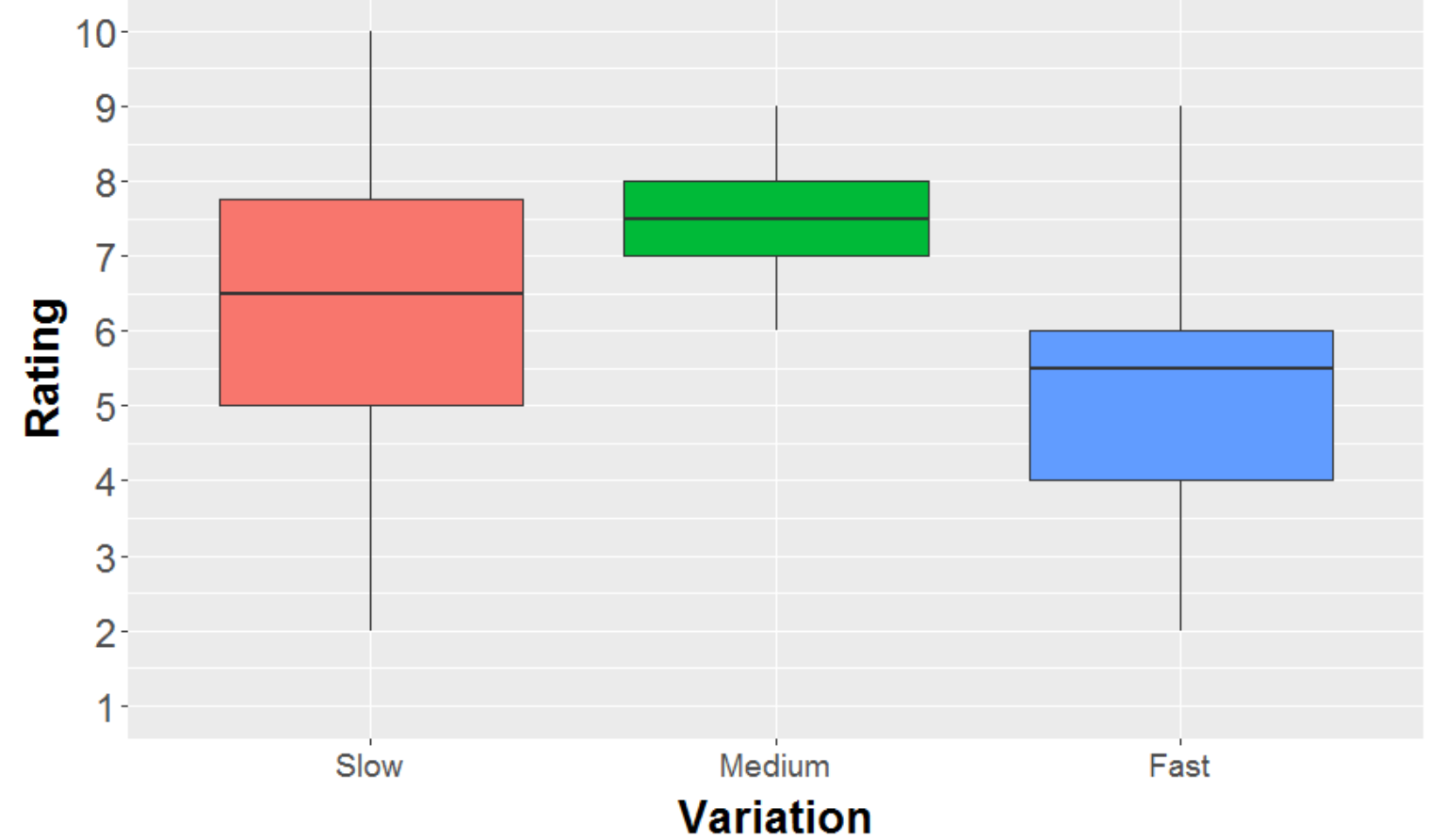

Figure 15: Preference ratings for different variations of the Animated technique. The medium speed was preferred more than the other two. The fast speed had the lowest ratings. 


\section{Preferences}

A set of questions about which technique or which variation of the technique participants preferred and felt more comfortable with were asked in the post-study experience questionnaire. Participant answered these questions by ranking each technique or variation of the technique on a scale from 1 to 10 .

The preference ratings were usually very close to each other with the exception for the fast variation of the Animated technique and the variation with the most number of intermediate points for the Pulsed technique that got very low ratings. The same pattern was also true for the ratings done by the participants who tested all the three techniques in the combination study. Their preference ratings for the techniques were not that different (See figures 14-17).

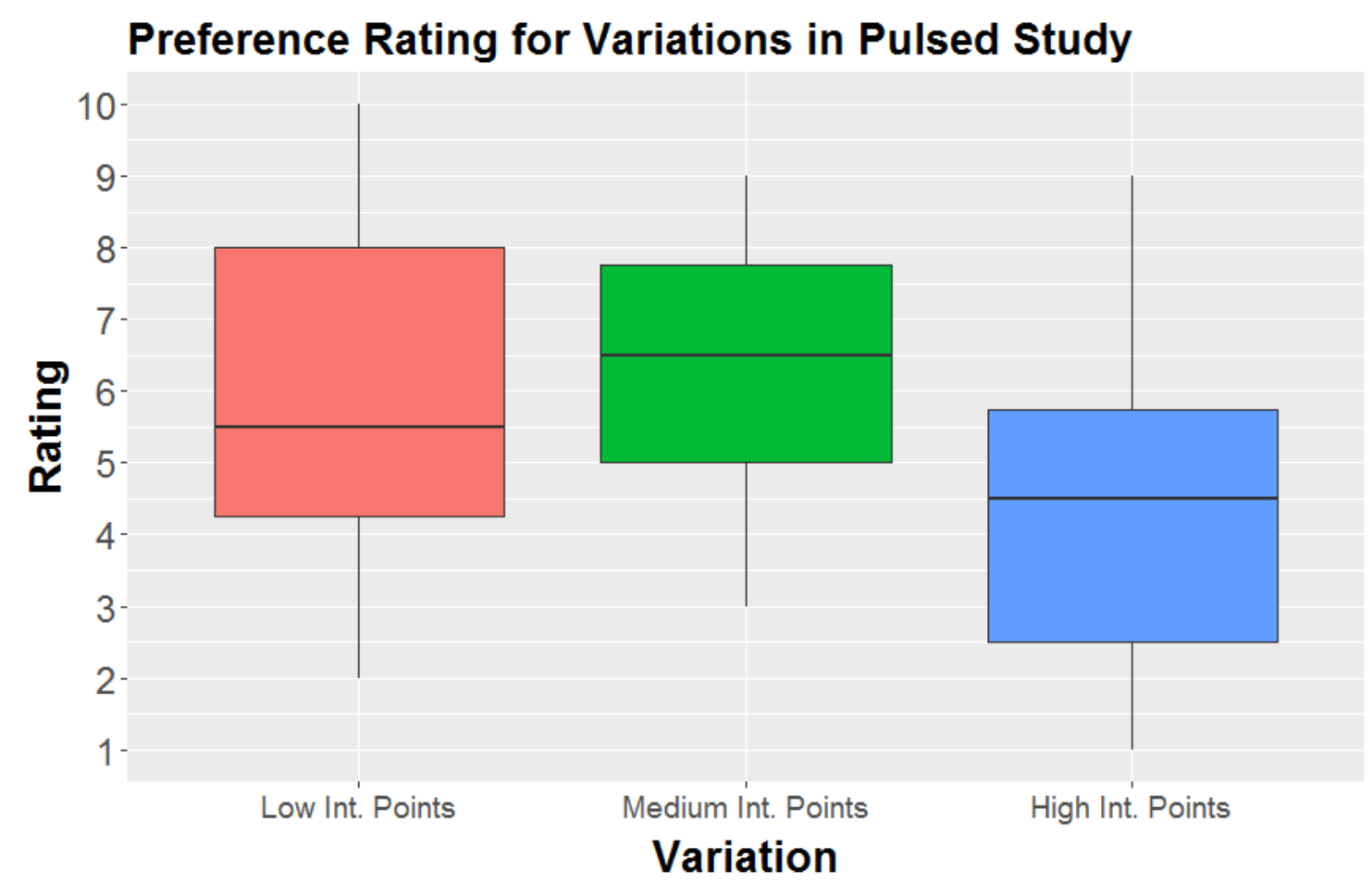

Figure 16: Preference ratings for different variations of the Pulsed technique. The variation with the high number of intermediate points was the one participants liked the least. 


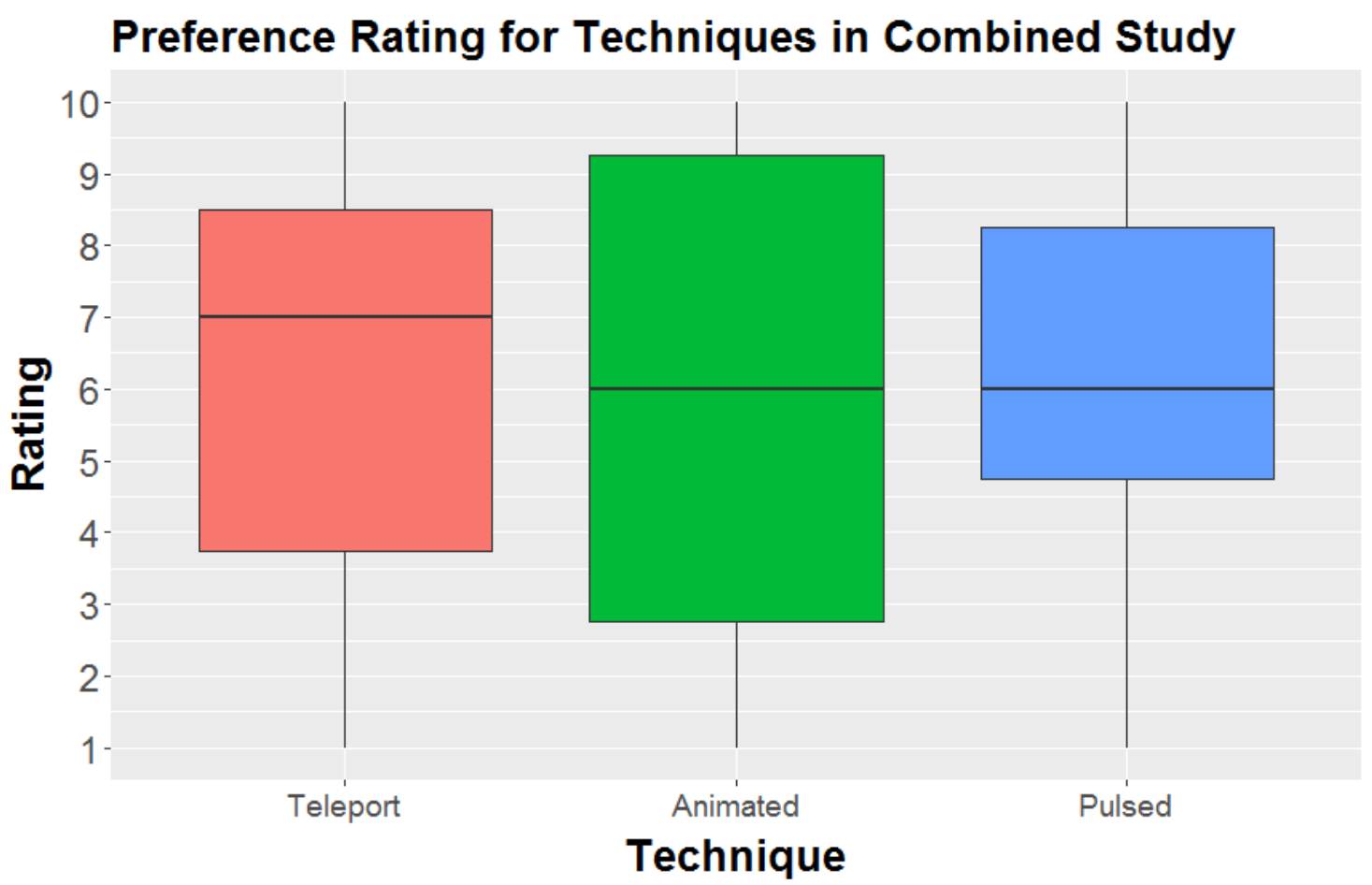

Figure 17: Preference ratings for different techniques in the combination study. There is no clear preference.

\section{Qualitative Feedback}

Participants provided feedback as part of the post-study experience questionnaire and the informal interview which was conducted at the very end of the experiment. The most common feedback was that rotating with the Animated technique caused a lot of discomfort and sickness. When testing the Animated technique, participants tried to fight the rotations by turning their head in the opposite direction which caused them nausea and they felt a bit of stomach awareness. The following are a few representative quotes about rotating using the Animated technique:

"Fast rotations gave me a high degree of dizziness."

"Rotation is weird because you inherently want to try to fight it."

"I got really dizzy during the rotations along the standing axis, and that disrupted my concentration and made things really difficult." 
In regards to the different scenes participants experienced in the study, participants gave varying comments. Some liked the fort scene because its objects had more color contrast, which may have made the task easier for them. They also mentioned that the landmarks in the fort were more distinct, and they were more confident when using them to remember where the disappeared barrel was after scene transitions. On the other hand, some participants preferred the Viking village stating that the larger area and the larger number of landmarks made the task easier. Also, they mentioned that since the village was not as bright as the fort, they felt more comfortable in that environment. Some of them even said that the bright colors in the fort scene hurt they eyes. 


\section{CHAPTER VI}

\section{DISCUSSION}

The study results showed that the errors for the spatial awareness task were relatively low for all the three scene transition techniques. That is also true for the sickness caused by the techniques. With the exception for rotating with the Animated technique, none of the other combinations of movement types and techniques caused any major sickness symptoms that really bothered the participants. Therefore, all of these scene transition techniques could be used in a 360-movie. Depending on what the goal of the director or the designer is in a specific situation, they can choose one of these techniques for the scene transition and camera movement to achieve the best results.

If the spatial orientation and awareness are the most important concerns in a scene, the Animated technique could be advantageous since the viewer will be able to keep track of the changes in their own positional state and the state of objects. The only exception is when the viewer should be rotated. Based on our findings and the participant feedback, the sickness caused by rotation with the Animated technique was not only is discomforting for the VR experience, but it also caused a loss of concentration that could be problematic when the goal is to maximize the spatial awareness.

If a comfortable VR experience with minimized dizziness and motion sickness is the goal, using the Teleportation technique is the best option. Both the instant and fade-to-black variations did not cause any significant sickness, and although Teleportation was not the perfect choice for maintaining spatial awareness when translating, its results for spatial awareness when rotating were very close to that of Animated and even better in some cases. 
Pulsed proved that it is actually a middle ground for Animated and Teleport. It had better results than Teleport for spatial awareness but it caused more sickness, and it had better results than Animated for the sickness but it was not as good in the spatial awareness tasks. Therefore, we believe that the Pulsed technique can be useful in some scenarios where the goal is for the viewer to be able to keep track of changes in the scene while not getting sick by the movements and transitions. In a long 360-movie, it is a good idea to use a combination of all the different techniques based on the scene and the situation the viewer is in.

In regards to the scenes and environments, based on the participants' feedback and the results, we found it is important for the scene to have clear and distinct landmarks to help the viewer maintain spatial awareness. We also found that in longer VR experiences, the use of bright colors might boost eyestrain in the viewers. 


\section{CHAPTER VII}

\section{CONCLUSION}

Our research explored different scene transition techniques and their variations in a 360movie and compared them with respect to the spatial awareness and sickness. The three techniques we studied were Teleportation with two different fade techniques, Animated Interpolation with three different speeds, and Pulsed Interpolation with three different numbers of intermediate points.

Our evaluation found that the relative error for the spatial awareness task and the sickness ratings were relatively low for all the scene transition techniques. The Animated technique proved to be the overall best for spatial awareness and the Teleport technique caused the least

sickness. The results for the Pulsed technique showed it was better than both the other techniques when it came to their weaknesses (relatively poor spatial awareness for Teleport and relatively high sickness for Animated), but it was worse than both of them when it came to their strengths (low sickness for Teleport and good spatial awareness for Animated). 


\section{REFERENCES}

[1] S. Beckhaus, K. J. Blom, and M. Haringer. Intuitive, hands-free travel interfaces for virtual environments. New Directions in 3D User Interfaces Workshop of IEEE VR, 2005. 1

[2] B. Bolte, F. Steinicke, and G. Bruder. The jumper metaphor: an effective navigation technique for immersive display setups. Proceedings of Virtual Reality International Conference, 2011. $1,2.1$

[3] D. A. Bowman, E. T. Davis, L. F. Hodges, and A. N. Badre. Maintaining spatial orientation during travel in an immersive virtual environment. Presence: Teleoperators and Virtual Environments, 8.6, 1999. 1, 2.1

[4] D. A. Bowman, D. Koller, and L. F. Hodges. Travel in immersive virtual environments: An evaluation of viewpoint motion control techniques. Virtual Reality Annual International Symposium, 1997. 1

[5] D. A. Bowman, D. Koller, and L. F. Hodges. A methodology for the evaluation of travel techniques for immersive virtual environments. Virtual reality, 3.2:120-131, 1998. 1, 2.1

[6] G. Bruder, F. Steinicke, and K. H. Hinrichs. Arch-explore: A natural user interface for immersive architectural walkthroughs. IEEE Symposium, pages 75-82, 2009. 1, 2.1

[7] S. S. Chance, F. Gaunet, A. C. Beall, and J. M. Loomis. Locomotion mode affects the updating of objects encountered during travel: The contribution of vestibular and proprioceptive inputs to path integration. Presence, 7.2:168-178, 1998. 1, 2.1

[8] S. Freitag, D. Rausch, and T. Kuhlen. Reorientation in virtual environments using interactive portals. IEEE Symposium, 2014. 1, 2.1

[9] Google spotlight stories. http://atap.google.com/spotlight-stories/. Accessed: 2016-11-27. 1

[10] R. S. Kennedy, N. E. Lane, K. S. Berbaum, and M. G. Lilienthal. Simulator sickness questionnaire: An enhanced method for quantifying simulator sickness. The international journal of aviation psychology, 1993.

[11] Oculus story studio. https://storystudio.oculus.com/en-us/. Accessed: 2016-11-27. 1 
[12] R. A. Ruddle and S. Lessels. The benefits of using a walking interface to navigate virtual environments. ACM Transactions on Computer- Human Interaction (TOCHI), 16.1:5, 2009. 1, 2.1

[13] E. A. Suma, S. Babu, and L. F. Hodges. Comparison of travel techniques in a complex, multi-level 3d environment. IEEE Symposium on 3D User Interfaces, 2007. 1, 2.1

[14] B. Tomlinson, B. Blumberg, and D. Nain. Expressive autonomous cinematography for interactive virtual environments. Proceedings of the fourth international conference on Autonomous agents, 2000. 2.1

[15] M. Usoh, K. Arthur, M. C. Whitton, R. Bastos, A. Steed, M. Slater, and F. P. Brooks Jr. Walking; walking-in-place; flying, in virtual environments. Proceedings of the 26th annual conference on Computer graphics and interactive techniques, 1999. 1, 2.1

[16] K. Vasylevska and H. Kaufmann. Influence of vertical navigation metaphors on presence. Proceedings of 15th International Conference on Presence, 2014. 2.1

[17] B. G. Witmer, J. H. Bailey, B. W. Knerr, and K. C. Parsons. Virtual spaces and real world places: transfer of route knowledge. International Journal of Human-Computer Studies, 45.4:413-428, 1996. 1 